\title{
Análisis de la opinión de usuarios sobre calidad percibida y satisfacción con hospitales públicos: Estudio de caso desde la perspectiva de la aplicación del marketing
}

\author{
Jorge Riveros $\mathrm{S}^{1 \mathrm{a}}$, Carmen Berné $\mathbf{M}^{2,3, b}$. \\ An enquiry on client opinions about \\ quality of care in a public hospital
}

Background: The level of satisfaction of patients with the services provided by public hospitals is becoming an important issue for health authorities. Aim: To analyze an enquiry applied to patients, about the level of satisfaction with the service provided by a public regional hospital in Chile. Material and methods: An enquiry was applied to 150 ambulatory ( $76 \%$ women) and 50 hospitalized patients ( $56 \%$ women). It included 28 questions about management aspects of health care and 20 questions about client satisfaction. Data was analyzed using factorial analysis and multiple regressions. Results: The manners of hospital personnel were the factor with the highest impact on the perception of patients about global quality of services. Attention timing was the most influential aspect on global satisfaction. Conclusions: There results confirm those from abroad and can orient an adequate management of public hospital (Rev Méd Chile 2007; 135: 862-70).

(Key words: Hospitals, public; Patient care management; Quality of health care)

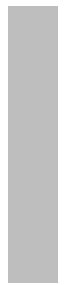

\begin{abstract}
Recibido el 26 de septiembre, 2006. Aceptado el 25 de enero, 2007.
Este trabajo de investigación cuenta con el patrocinio y financiamiento de la Dirección de Investigación de la Universidad Austral de Chile (Proyecto D.I.D. S 2003-68), no teniendo influencia en el diseño del estudio ni en el tratamiento de los datos de investigación.

${ }^{1}$ Instituto de Administración, Universidad Austral de Chile. ${ }^{2}$ Departamento de Economía y Dirección de Empresas, Universidad de Zaragoza, España. ${ }^{3}$ Grupo de Investigación CREVALOR. angeniero Comercial, candidato a doctorado Universidad de Zaragoza (España).

bDoctora en Ciencias Económicas y Empresariales
\end{abstract}

$E^{1}$ interés de este estudio se explica por la creciente necesidad de conocer las percepciones de calidad y el nivel de satisfacción que pacientes y usuarios, en general, obtienen de los

Correspondencia a: Jorge Riveros S. Casilla de correo 567, Valdivia, Chile. Fono fax: 63-221679. E mail: jriveros@uach.cl servicios de salud. Este conocimiento está siendo cada vez más relevante para la formulación y evaluación de políticas sociales, y, en particular, de políticas públicas de salud, aceptando que un mayor grado de satisfacción de los usuarios, asociado a percepciones de mayor calidad, es un resultado social deseable y deseado por la gestión hospitalaria pública y consecuente con la aplica- 
ción del marketing en este sector, que se ha estado analizando ${ }^{1}$.

Bajo estas consideraciones, este trabajo tiene como propósito identificar los componentes principales de gestión orientados al usuario, que tienen incidencia en sus percepciones de calidad; como también los elementos del servicio que influirían en su satisfacción global. Estos resultados deben proporcionar una base útil para la puesta en marcha de aspectos de gestión -en especial asociados al marketing- que mejoren la calidad de atención al usuario del centro hospitalario y consigan elevar su nivel de satisfacción con el mismo.

El punto de partida respecto a la calidad de servicio, es que ésta depende del ajuste entre la gestión de dicho servicio (y su orientación) y las expectativas del cliente o usuario; lo que supone en definitiva, una comparación entre el servicio esperado y el servicio recibido ${ }^{2}$.

Los estudios aplicados al sector han mostrado que los pacientes dan menor importancia a atributos que son difíciles de valorar por su elevado grado de intangibilidad; y confían más en atributos de experiencia para juzgar el servicio ${ }^{3}$. Por tanto, el énfasis principal de la evaluación debe estar en aspectos del proceso de entrega del mismo (Figura 1$)^{4}$.

Con respecto a la medición de la calidad percibida, las propuestas más importantes han sido la escala SERVQUAL ${ }^{5}$, que recoge informaciones sobre percepciones y expectativas para cada atributo, y la escala SERVPERF ${ }^{6}$ que recoge solamente percepciones, y que se ha demostrado posteriormente como más práctica y efectiva para esta medición, estableciéndose, además, que el sector de aplicación es clave para la identificación de las dimensiones del constructo propuesto ${ }^{7}$.

Por otra parte, algunos autores ${ }^{8}$ consideran además, que la calidad de servicio percibida es un antecedente de la satisfacción. Al igual que la calidad, la satisfacción es un concepto complejo y multidimensional compuesto de varios factores, basados en diferentes experiencias con los empleados que prestan el servicio y con otros elementos tangibles (comodidades de infraestructura, alimentación, etc.) e intangibles ${ }^{9}$; como entrega de información, grado de accesibilidad, nivel de burocracia, humanización del servicio, atención prestada a problemas psicosociales, etc ${ }^{10}$.

El concepto satisfacción se define como una evaluación postconsumo o postuso, fuente de desarrollo y cambio de actitudes hacia el objeto de consumo o uso, y que es el resultado de procesos psicosociales de carácter cognitivo y afectivo ${ }^{11}$, entre ellos, la propia percepción de calidad ${ }^{12}$.

Fitzpatrick ${ }^{13}$ afirma que el grado de satisfacción se relaciona con la efectividad de la atención médica; ya

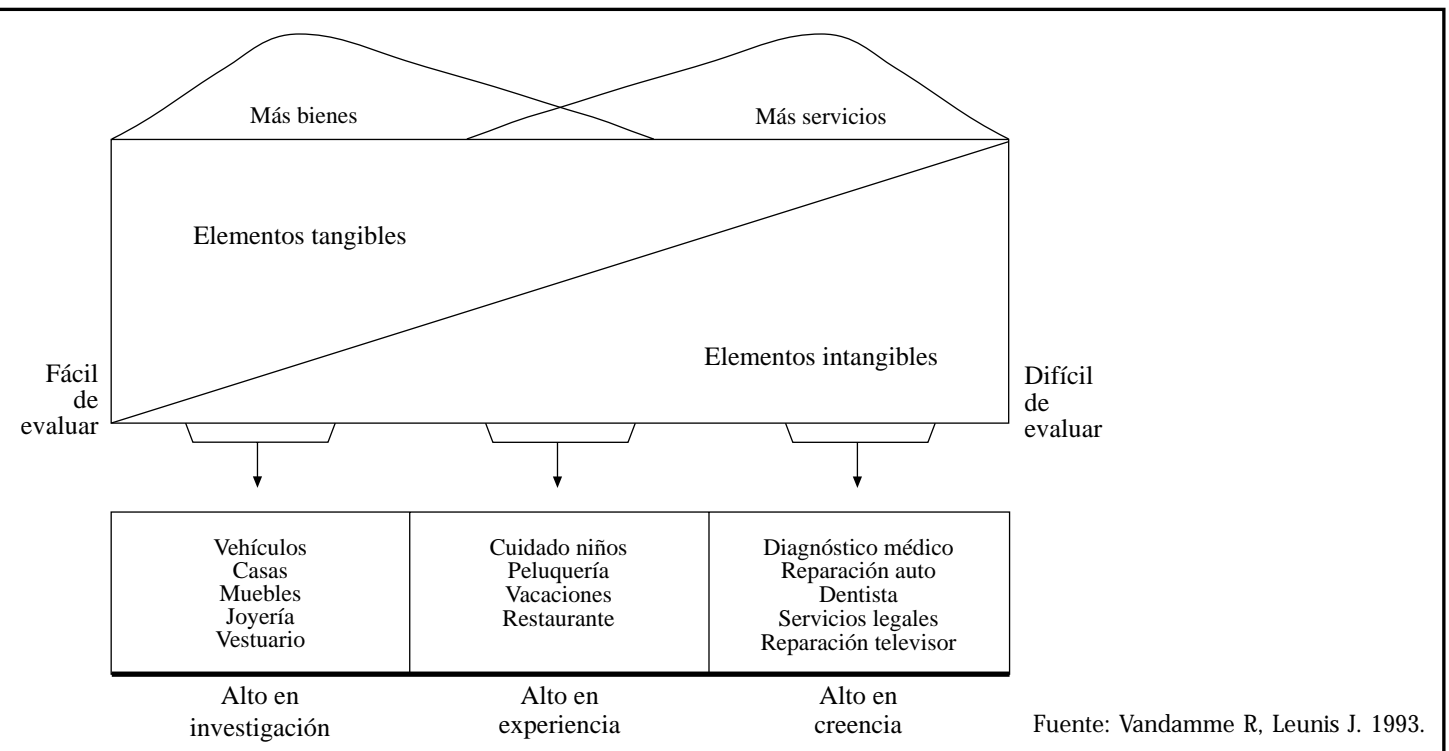

Figura 1. Evaluación de productos y servicios. 
que los pacientes con bajos grados de satisfacción regresan menos a sus controles, tienen menor adherencia a sus tratamientos y presentan pobres resultados en salud. De manera que mayores niveles de satisfacción con el servicio podnín determinar una mayor efectividad de la atención prestada.

Por tanto el tema es, cómo la calidad y la satisfacción y su relación, pueden asegurar una mayor eficacia y productividad sanitaria de los recursos que la sociedad entrega a los centros hospitalarios cuyos presupuestos concentran, actualmente en Chile, más de $80 \%$ del gasto público en salud ${ }^{14}$.

\section{MATERIAL Y MÉTOdO}

El análisis empírico se desarrolla a través de la aplicación de una encuesta, diseñada para tal efecto, a una muestra de usuarios de un hospital del sur de Chile. Considerando la amplia cobertura poblacional que atiende este establecimiento, se realizó un muestreo por cuotas, teniendo en cuenta el tipo y porcentaje de atención cubierta por edades de los usuarios/pacientes, a partir de información proporcionada por su dirección. La muestra se conformó con 200 pacientes/usuarios, hombres y mujeres mayores de 15 años, que acababan de ser atendidos o que se encontraban hospitalizados durante el período en que se aplicó la encuesta y cuya composición se puede visualizar en la Tabla 1.

Tanto del consultorio ambulatorio externo como de los servicios de hospitalización, se consideraron las especialidades que poseían mayores prestaciones dentro de un año normal; así como también, todo lo referente al entorno social de la atención (relación entre personal funcionario y usuario), entorno físico (infraestructura) y procesos administrativos del servicio.

El cuestionario consideraba dos grandes bloques temáticos, planteados sobre una escala Likert de siete puntos, dirigidos a obtener información sobre percepciones de los encuestados. El primero consideraba 28 afirmaciones, de las que se recogía desde «Nunca» (valor 1), hasta «iempre» (valor 7), la opinión del usuario sobre la ocurrencia de aspectos de gestión orientados hacia la atención de sus necesidades de salud. El otro consideraba 20 afirmaciones, en las que se pretendía medir el grado de satisfacción del usuario, desde «Completamente Insatisfecho» (valor 1) hasta «Completamente Satisfecho» (valor 7), con distintos elementos del servicio. Una pregunta más en cada bloque, se dirigía a obtener una opinión global sobre la percepción de calidad de la atención y sobre la satisfacción global con el servicio recibido.

La encuesta se aplicó durante los meses de septiembre y octubre de 2003. Para el análisis de los datos obtenidos, se utilizó el programa SPSS 11.5. Se realizó, al igual que en casos similares ${ }^{15}$, análisis de fiabilidad y análisis factorial exploratorio, con método de extracción de componentes principales y rotación Varimax, para los dos aspectos en estudio. Posteriormente, análisis de regresión múltiple permitieron determinar el impacto de los factores extraídos sobre la calidad percibida y la satisfacción global de los usuarios.

\section{RESUlTADOs}

Del análisis de fiabilidad aplicado al conjunto de indicadores de gestión, resultó la eliminación de dos de ellos, alcanzando un Alpha de Cronbach de 0.9292. Para el caso de la satisfacción con distintos elementos del servicio, se introdujeron 19 ítems (después de eliminar uno para incrementar fiabilidad), que arrojaron un Alpha de 0,9040.

Factorial exploratorio de aspectos de gestión orientados al usuario. El análisis arroja seis factores que explican

Tabla 1. Composición de la muestra de usuarios del hospital

\begin{tabular}{|lcccccc|}
\hline Composición de la muestra & Hombres & Mujeres & 15 a 24 años & 25 a 44 años & 45 a 64 años & 65 años y más \\
\hline 150 usuarios & 36 & 114 & 29 & 53 & 39 & 29 \\
Ambulatorios (75\%) & $(24 \%)$ & $(76 \%)$ & $(19 \%)$ & $(36 \%)$ & $(26 \%)$ & $(19 \%)$ \\
50 usuarios & 22 & 28 & 9 & 18 & 13 & 10 \\
Hospitalizados (25\%) & $(44 \%)$ & $(56 \%)$ & $(18 \%)$ & $(36 \%)$ & $(26 \%)$ & $(20 \%)$ \\
\hline
\end{tabular}

Fuente: Elaboración propia. 
prácticamente $70 \%$ de la varianza total (Tabla 2). La composición de los factores se encuentra en Tabla 3.
El Factor 1 podría identificarse con la «estión de informaciones y reclamos». Este fue el que

Tabla 2. Análisis factorial de aspectos de gestión orientados al usuario que incidirían en calidad percibida

\begin{tabular}{|lccc|}
\hline Componentes principales & Varianza total & \% Varianza & \% Varianza acumulada \\
\hline Factor 1 & 4.756 & 18.292 & 18.292 \\
Factor 2 & 3.358 & 12.917 & 31.209 \\
Factor 3 & 2.918 & 11.221 & 42.430 \\
Factor 4 & 2.881 & 11.079 & 53.509 \\
Factor 5 & 2.432 & 9.355 & 62.864 \\
Factor 6 & 1.598 & 6.147 & 69.011 \\
\hline
\end{tabular}

Fuente: Elaborado en SPSS v. 11.5 (Matriz de componentes rotados) (Normalización Varimax con Kaiser)

Tabla 3. C argas factoriales y análisis de fiabilidad sobre aspectos de gestión orientados al usuario que incidirían en calidad percibida*

\begin{tabular}{|c|c|c|c|c|c|c|}
\hline Variables de análisis & $\begin{array}{r}\text { Factor } \\
1\end{array}$ & $\begin{array}{r}\text { Factor } \\
2\end{array}$ & $\begin{array}{r}\text { Factor } \\
3\end{array}$ & $\begin{array}{r}\text { Factor } \\
4 \\
\end{array}$ & $\begin{array}{r}\text { Factor } \\
5 \\
\end{array}$ & $\begin{array}{r}\text { Factor } \\
6 \\
\end{array}$ \\
\hline Trato personal de informaciones (X24) & 0,855 & & & & & \\
\hline Disposición atender reclamos (X25) & 0,849 & & & & & \\
\hline Informaciones en lugar visible (X23) & 0,820 & & & & & \\
\hline Preocupación por resolver quejas (X26) & 0,766 & & & & & \\
\hline Medios apropiados ofic reclamos (X22) & 0,742 & & & & & \\
\hline Información derechos y deberes usuarios (X27) & 0,643 & & & & & \\
\hline Personal usa inform. confidencial (X10) & & 0,823 & & & & \\
\hline Personal con inform. caso personal (X9) & & 0,712 & & & & \\
\hline Comunicación permanente con el usuario (X11) & & 0,626 & & & & \\
\hline Mantener interés estado de salud usuario (X12) & & 0,577 & & & & \\
\hline Trabajo coordinado de servicios (X13) & & 0,460 & & & & \\
\hline Trato de médicos (X16) & & & 0,784 & & & \\
\hline Conocim. funcionarios administ. (X20) & & & 0,731 & & & \\
\hline Trato funcionarios administ. (X21) & 0,487 & & 0,629 & & & \\
\hline Trato enfermeras y auxiliares (X19) & & & 0,569 & 0,566 & & \\
\hline Compromiso y disposición funcionarios (X14) & & & 0,457 & & & \\
\hline Funcionamiento hospital (X2) & & & & 0,756 & & \\
\hline Funcionarios comprometidos (X3) & & & & 0,698 & & \\
\hline Capacidades enfermeras y auxiliares (X18) & & & 0,473 & 0,621 & & \\
\hline Capacidades y habilidades de médicos (X15) & & & & 0,504 & & \\
\hline Todos los servicios comprometidos (X4) & & & & & 0,762 & \\
\hline Contacto permanente con usuarios (X7) & & & 0,453 & & 0,550 & \\
\hline Preocupación real del hospital (X1) & & & & 0,454 & 0,533 & \\
\hline Educación sobre el servicio al cual acudir (X28) & & & & & 0,516 & \\
\hline Hospital brinda solución integral (X5) & & & & & 0,466 & \\
\hline Contacto directo con persona (X8) & & & & & & 0,840 \\
\hline $\begin{array}{l}\text { Alpha de Cronbach de cada factor } \\
\text { (con variables finales de cada factor) }\end{array}$ & 0,8952 & 0,7931 & 0,8541 & 0,7764 & 0,6956 & -- \\
\hline
\end{tabular}

* Para la constitución de las variables de los distintos factores, se consideró el criterio de 0,450 de carga factorial mínima. Fuente: Elaboración propia en base a SPSS v.11.5. 
explicó un mayor porcentaje de varianza (18\%), y define, como ítems de mayor peso, el trato ofrecido por el personal de la oficina de informaciones y reclamos, la disposición a la atención de sugerencias y una conveniente ubicación de la oficina.

El Factor 2 está relacionado con la «Gestión de datos del usuario/paciente». Los ítems más destacados son los enunciados sobre el buen uso de la información individual recogida del usuario/paciente, y la posesión por parte del personal de atención; de una información conveniente, completa y actualizada.

El Factor 3, se denomina «rato prestado por el personal», identificándose con ítems relativos a la relación entre el personal médico y administrativo con el usuario/paciente.

Estos tres factores se encuentran ligados estrechamente a políticas de marketing y en especial a uno de sus enfoques más recientes, el marketing relacional.

El Factor 4 corresponde a «Gestión de capacidades y compromisos orientados al usuario», que considera la orientación del funcionamiento del hospital y de las capacidades del personal médico y paramédico hacia la satisfacción de las necesidades del usuario y el compromiso de los funcionarios con el mismo objetivo. Este factor también se encuentra estrechamente relacionado con políticas de marketing y en especial con el enfoque de orientación al cliente 0 usuario ${ }^{16}$.

Los últimos factores ( 5 y 6 ), que también se encuentran ligados a este último enfoque de marketing, explican un menor porcentaje de la varianza: «Gestión de información y coordinación entre servicios» y «Gestión personalizada al usuario», que tiene entidad propia con un único ítem.
Factorial exploratorio de elementos del servicio. El análisis factorial aplicado extrae en este caso un total de seis factores que explican $73,5 \%$ de la varianza (Tabla 4) (ver composición de factores en Tabla 5).

El Factor 1 corresponde a aspectos de «Mantenimiento y limpieza del hospital»y y explica $18,4 \%$ de la varianza.

El Factor 2 está relacionado con «iempos de atención», tanto para la atención médica propiamente tal, como para la espera y el ingreso a hospitalización.

El Factor 3 está relacionado con «Comodidades de salas de espera y señalizaciones del recinto».

El Factor 4 se refiere a aspectos de «nfraestructura del hospital».

Finalmente, los Factores 5 y 6, que representan un menor porcentaje de varianza explicada, consideran «Comodidades de salas de hospitalización» (incluyen mobiliario y días de visita de familiares) y «Asistencia social y psicológica».

Análisis de regresión: la calidad percibida en función de factores de gestión. Una vez obtenidos los factores relativos a una gestión orientada a resolver necesidades de salud y comprobada su fiabilidad (Tabla 5), se procedió a determinar sus potenciales efectos sobre el nivel de calidad global percibido por los usuarios.

Para ello, se utilizó análisis de regresión múltiple, método stepwise, siendo la variable dependiente la spercepción de calidad global de la atención»y las variables explicativas, los factores extraídos.

Los resultados obtenidos indican que los cinco primeros factores ejercían un efecto positivo y estadísticamente significativo $(\mathrm{p}<0,01)$ sobre la variable dependiente. Como puede observarse en

Tabla 4. Análisis factorial de elementos del servicio que incidirían en la satisfacción del usuario

\begin{tabular}{|lccc|}
\hline Componentes principales & Varianza Total & \% Varianza & \% Varianza acumulada \\
\hline Factor 1 & 3.494 & 18.387 & 18.387 \\
Factor 2 & 3.025 & 15.922 & 34.309 \\
Factor 3 & 2.153 & 11.333 & 45.643 \\
Factor 4 & 1.909 & 10.047 & 55.690 \\
Factor 5 & 1.773 & 9.331 & 65.021 \\
Factor 6 & 1.607 & 8.455 & 73.476 \\
\hline
\end{tabular}

Fuente: Elaborado en SPSS v.11.5 (matriz de componentes rotados) (Normalización Varimax con Kaiser). 
Tabla 5. C argas factoriales y análisis de fiabilidad de elementos del servicio que incidirían en la satisfacción global del usuario*

\begin{tabular}{|c|c|c|c|c|c|c|}
\hline Variables de análisis & $\begin{array}{r}\text { Factor } \\
1\end{array}$ & $\begin{array}{r}\text { Factor } \\
2\end{array}$ & $\begin{array}{r}\text { Factor } \\
3\end{array}$ & $\begin{array}{r}\text { Factor } \\
4\end{array}$ & $\begin{array}{r}\text { Factor } \\
5\end{array}$ & $\begin{array}{r}\text { Factor } \\
6\end{array}$ \\
\hline Comodidad, limpieza, mantención baños (X39) & 0,794 & & & & & \\
\hline Número y ubicación baños usuarios (X38) & 0,752 & & & & & \\
\hline Comodidad camas y ropa de cama (X34) & 0,731 & & & & & \\
\hline Limpieza salas hospital, espera, pasillos (X36) & 0,703 & & & & & \\
\hline Alimentación período hospitalización (X37) & 0,622 & & & & & \\
\hline Proceso tramitación horas médicas (X42) & & 0,847 & & & & \\
\hline Ingreso a hospitalización (X43) & & 0,827 & & & & \\
\hline Tiempo de espera para atención (X44) & & 0,814 & & & & \\
\hline Tiempo dedicado a la atención personal (X45) & & 0,739 & & & & \\
\hline Comodidad cantidad asientos sala espera (X32) & & & 0,809 & & & \\
\hline Comodidades salas de espera (X31) & & & 0,783 & & & \\
\hline Señalizaciones recinto (X30) & & & 0,648 & & & \\
\hline Mantenc. instalaciones atención segur (X41) & & & & 0,819 & & \\
\hline Comodidad y facilidades circulación (X40) & & & & 0,781 & & \\
\hline Días de visita familiares (X46) & & & & & 0,663 & \\
\hline Comodidad muebles sala hospitalizados (X35) & 0,582 & & & & 0,613 & \\
\hline Comodidades salas hospitalizados (X33) & & & & & 0,600 & \\
\hline Asistencia social ofrecida (X47) & & & & & & 0,829 \\
\hline Asistencia espiritual y psicológica (X48) & & & & & & 0,764 \\
\hline $\begin{array}{l}\text { Alpha de Cronbach de cada factor } \\
\text { (con variables finales de cada factor) }\end{array}$ & 0,8277 & 0,8730 & 0,7559 & 0,7737 & 0,6697 & 0,5969 \\
\hline
\end{tabular}

*Para la constitución de las variables de los distintos factores, se consideró el criterio de 0,450 de carga factorial mínima. Fuente: Elaboración propia en base a SPSS v.11.5.

la Tabla 6, los aspectos relacionados con el trato otorgado por el personal hospitalario fueron los que ejercían una mayor influencia en el nivel de calidad percibido. El segundo factor en importancia resultó ser la gestión de capacidades y compromisos orientados al usuario. A estos factores, les siguieron los de gestión de información y coordinación entre servicios, gestión de informaciones y reclamos, y finalmente, gestión de datos de usuarios/pacientes. Estas variables, que se encuentran relacionadas con la aplicación de políticas de marketing y en especial con sus enfoques más avanzados, fueron capaces de explicar en gran medida la percepción global de los usuarios sobre la calidad del hospital, ya que el $\mathrm{R}^{2}$ ajustado fue $58,2 \%$.
Análisis de regresión: satisfacción global en función de factores del servicio. De igual forma, se procedió a determinar los potenciales efectos de los distintos factores del servicio (variables independientes) sobre el nivel de satisfacción global del usuario (variable dependiente).

Los resultados (Tabla 7) indican que los seis factores obtenidos ejercen un efecto positivo y estadísticamente significativo $(p<0,05)$ sobre el nivel de satisfacción de los usuarios con el servicio ofrecido por el hospital.

La comparación de los coeficientes estimados estandarizados, permite comprobar que la satisfacción con los tiempos de atención fue el factor que ejerció una mayor influencia sobre este nivel. A éste, le siguen las comodidades de salas de hospitalización (con un efecto algo menor de la 
Tabla 6. Análisis de regresión: Calidad percibida en función de factores de gestión orientados al usuario

\begin{tabular}{|c|c|c|c|c|}
\hline \multicolumn{2}{|c|}{$\begin{array}{l}\text { Variable dependiente: } \\
\text { Calidad global percibida por el usuario } \\
\text { Variables independientes }\end{array}$} & $\begin{array}{l}\text { Coeficiente Beta } \\
\text { estandarizado }\end{array}$ & t value & Significatividad \\
\hline Constante & & & 66.811 & 0,000 \\
\hline Factor 3: & Trato prestado por el personal del hospital & 0,510 & 8.121 & 0,000 \\
\hline Factor 4: & $\begin{array}{l}\text { Gestión de capacidades y compromisos } \\
\text { orientados al usuario }\end{array}$ & 0,381 & 6.067 & 0,000 \\
\hline Factor 5: & $\begin{array}{l}\text { Gestión de información y coordinación } \\
\text { entre servicios }\end{array}$ & 0,284 & 4.513 & 0,000 \\
\hline Factor 1: & Gestión de informaciones y reclamos & 0,275 & 4.371 & 0,000 \\
\hline Factor 2: & $\begin{array}{l}\text { Gestión de datos del usuario/paciente } \\
\mathrm{R}^{2}: 0,601 ; \mathrm{R}^{2} \text { ajustado: 0,582 }\end{array}$ & 0,200 & 3.185 & 0,000 \\
\hline
\end{tabular}

Fuente: Elaboración propia a partir de los resultados obtenidos en SPSS v. 11.5. Análisis de regresión con método stepwise.

Tabla 7. Análisis de regresión: Satisfacción global del usuario en función de elementos del servicio

\begin{tabular}{|c|c|c|c|c|}
\hline \multicolumn{2}{|c|}{$\begin{array}{l}\text { Variable dependiente: } \\
\text { Satisfacción global con el servicio } \\
\text { Variables independientes }\end{array}$} & $\begin{array}{l}\text { Coeficiente Beta } \\
\text { estandarizado }\end{array}$ & t value & Significatividad \\
\hline \multirow{4}{*}{$\begin{array}{l}\text { Constante } \\
\text { Factor 2: } \\
\text { Factor 5: }\end{array}$} & & & 83.313 & 0,000 \\
\hline & Satisfacción con tiempos de atención & 0,451 & 6.854 & 0,000 \\
\hline & Satisfacción con comodidades de salas & & & \\
\hline & de hospitalización (incluye días visita familiares) & 0,273 & 4.154 & 0,000 \\
\hline \multirow{3}{*}{$\begin{array}{l}\text { Factor 1: } \\
\text { Factor 6: } \\
\text { Factor 3: }\end{array}$} & Satisfacción con mantenimiento y limpieza del hospital & 0,215 & 3.260 & 0,001 \\
\hline & Satisfacción con asistencia social y psicológica & 0,189 & 2.878 & 0,005 \\
\hline & $\begin{array}{l}\text { Satisfacción con comodidades de salas } \\
\text { de espera y señalizaciones del recinto }\end{array}$ & 0,182 & 2.767 & 0,006 \\
\hline Factor 4: & $\begin{array}{l}\text { Satisfacción con infraestructura del hospital } \\
R^{2}: 0,411 ; R^{2} \text { ajustado: } 0,385\end{array}$ & 0,131 & 1.992 & 0,048 \\
\hline
\end{tabular}

Fuente: Elaboración propia a partir de los resultados obtenidos en SPSS v.11.5. Análisis de regresión con método stepwise.

mitad que el anterior) y el mantenimiento y limpieza del hospital. Un efecto menor fue el que provoca el servicio de asistencia social y psicológica, al que le siguieron el ofrecido por las comodidades de salas de espera y señalizaciones del recinto $\mathrm{y}$, finalmente, aspectos relacionados con infraestructura del establecimiento. Estas variables fueron capaces de explicar aproximadamente $40 \%$ del nivel global de satisfacción de los usuarios.

\section{DISCUSIÓN}

Este estudio no tiene antecedentes en la literatura y revela que la satisfacción de los clientes está estrechamente ligada a la consecución de objetivos organizacionales ${ }^{17}$. Dicho de otro modo, los procesos de gestión y su orientación han de someterse a evaluación y mejora continua con el objetivo de procurar la satisfacción de los clientes de la organización. 
Desde la perspectiva de los usuarios/pacientes de un hospital público, el análisis realizado ha identificado y validado, en términos iniciales, los factores de gestión orientados al usuario que inciden en sus percepciones de calidad, así como los factores específicos del servicio que influyen en su satisfacción global.

Varios de los factores de gestión que inciden en la percepción de calidad son aspectos relacionados con la aplicación del marketing -tanto desde el punto de vista tradicional de la disciplina, como de sus enfoques más avanzados (orientación al cliente o usuario y marketing relacional)-, lo cual viene a complementar los resultados obtenidos por Riveros y Bemé ${ }^{1}$ desde la perspectiva de los funcionarios.

Asimismo, se ha podido determinar que el trato otorgado por el personal del hospital es el que provoca un mayor efecto sobre la calidad percibida por el usuario/paciente. En segunda instancia influyen una gestión de capacidades y compromisos orientados al usuario y también, la gestión de información y coordinación entre los servicios internos.

Con respecto a la satisfacción de usuarios/ pacientes con distintos elementos del servicio, se señala que el factor que más influye es el relativo a los tiempos de atención. Es decir, una mejor percepción sobre la tramitación de horarios de consulta, ingreso y espera, proporciona en mayor medida que otros aspectos, una mayor satisfacción global con el servicio recibido. Esto, junto con comodidades de salas de hospitalización y mantenimiento y limpieza general, deben fortalecerse en orden a conseguir mejorar la satisfacción global de los usuarios.

El resultado confirma lo que ha sido descrito en encuestas nacionales realizadas en Estados Unidos de Norteamérica, Canadá y Reino Unido ${ }^{18}$, donde se manifestaba que los puntos más críticos de la evaluación de calidad, tienen que ver con aspectos de comunicación (trato) entre el paciente y el equipo que le brinda la atención (explicación del diagnóstico y tratamiento, indicaciones después del alta, signos con los cuales el paciente debe tener cuidado, etc.). Esta observación es importante porque, por un lado, viene a destacar el alto nivel de creencia que dificulta la evaluación del servicio y la necesidad de utilizar atributos de experiencia con el mismo para poder obtener un juicio más efectivo. Y por otro lado, surgen reticencias respecto al uso frecuente que se ha venido haciendo de estrategias orientadas a resolver sólo problemas de infraestructura para mejorar el grado de satisfacción de los pacientes/usuarios del sistema de salud. Aunque resolver estos problemas es ineludible, los resultados muestran que no se puede esperar que dichas estrategias logren una mejoría sustancial en la evaluación que los pacientes/usuarios hacen del sistema público de salud. Para el logro de esta meta, será fundamental mejorar el trato por parte del personal: médico, paramédico y funcionarios administrativos, así como administrar los tiempos de atención.

Aunque entendemos que este estudio proporciona conclusiones relevantes en relación al objetivo perseguido, la aplicación se ha probado para un caso concreto, lo que supone una limitación de la investigación que justifica la conveniencia de replicar el análisis en otros establecimientos hospitalarios.

A más corto plazo, la investigación futura se dirige al estudio de la relación causa-efecto entre los constructos: calidad percibida de la atención recibida y satisfacción global de los usuarios. La confirmación de los modelos de medida de estos constructos, mediante análisis factorial confirmatorio, y el uso de la metodología de ecuaciones estructurales son los pasos previstos para este desarrollo.

\section{Agradecimientos}

Reconocemos la labor desarrollada por los académicos de la Universidad Austral de Chile, miembros del equipo de investigación del Proyecto D.I.D., Sres. Juan Carlos Miranda C (Estadístico), Sra. Angélica Wenzel K. (Administrador Público), Sr. Enrique López R. (Administrador Público), Sr. Juan Carlos Bertoglio C. (Médico Cirujano) y Sra. María Cristina Torres (Enfermera y Matrona) quienes participaron en el diseño de la muestra y de la encuesta, en el análisis estadístico de la información, en asesoría técnica y administrativa y en distintos aportes a la realización del trabajo. 


\section{REFERENCIAS}

1. Rveros J, BerNe C. La Aplicación del marketing en hospitales públicos desde la perspectiva de los funcionarios: El caso de un hospital del sur de Chile. Rev Méd Chile 2006; 134: 353-60.

2. Parasuraman A, Zeithaml V, Berry L. Guidelines for Conducting Service Quality Research. Marketing Research 1990; 34-44.

3. Donabedian A. The Methods and Findings of Quality Assessment and Monitoring: An Illustrated Analysis. Michigan, IN.: Ann Arbor, 1985.

4. Vandamme R, Leunis J. Development of a Multipleitem Scale for Measuring Hospital Service Quality. International Journal of Service Industry Management 1993; 4: 30-49.

5. Parasuraman A, Zeithaml V, Berry L. SERVQuAL: A Multiple-Item Scale for Measuring Consumer Perceptions of Service Quality. Journal of Retailing 1988; 64: 12-40.

6. CRONIN J, TAYLOR S. SERVPERF versus SERVQUAL: Reconciling Performance-Based and PerceptionsMinus-Expectations Measurement of Service Quality. Journal of Marketing 1994; 58: 125-31.

7. Hernández R, Muñoz P, SANTos L, González O. Una propuesta general de análisis de la calidad y la satisfacción en el turismo rural español. En: http:/ / www.esade.es/cedit2003/pdfs/ ernandezrosamaria.pdf 2003. [Consultado el 4 de diciembre de 2006].

8. Bolton R, Drew J. A Multistage Model of Customer's Assessments of Service Quality and Value. Journal of Consumer Research 1991; 17: 375-84.

9. Marzo M, Pedraja M, Rivera P. Determinants of satisfaction with university courses. Quality in Higher Education 2005; 11: 239-49.
10. Alvarado R, Vera A. Evaluación de la satisfacción de usuarios del Sistema Nacional de Servicios de Salud. Revista Chilena de Salud Pública 2001; 5: 81-9.

11. Moraies V, Hernández A. Calidad y satisfacción en los servicios: conceptualización. Revista Digital 2004; 73: junio. [Disponible en http://www.efdeportes.com y consultada el 4 de diciembre de 2006].

12. BERNÉ C, MúGICA JM, YAGÜE MJ. La gestión estratégica y los conceptos de calidad percibida, satisfacción del cliente y lealtad. Economía Industrial 1996; 307: 63-74.

13. Fitzpatrick R. The assessement of patient satisfaction. En: Assessement and Evaluation of Health and Medical Care: A methods text. London, UK: Open University Press, 1997.

14. Gobierno de Chile. Hacia un nuevo modelo de gestión en salud 2002; junio. (Disponible en http:// www.saludyfu tu ro.cl/images/pdf/ gestion.pdf y consultada el 22 de marzo de 2006].

15. Dos Reis Rodríguez AJ, Raposo ML, Do Paco A. Estudio sobre la satisfacción de los usuarios de una unidad de salud. En: Actas de las II Jornadas Internacionales de Marketing Público y No Lucrativo. Zaragoza (España), 2003; 141-52.

16. Hoekstra J, Leefiang P, Wittink D. The Customer Concept: The Basis for a New Marketing Paradigm. Journal of Market-Focused Management 1999; 4: 43-76.

17. Agus A, KRISHNAN S, KadiR S. The structural impact of total quality management on financial performance relative to competitors through customer satisfaction: a study of Malaysian manufacturing companies. Total Quality Management 2000; 11: 808-19.

18. Bruster S, Jarman B, Bosanquet N, Weston D, Erens R, DelBanco T. National survey of hospital patients. En: BMJ (Clinical Research ed.) 1994; 309: 1542-6. 